\title{
El perfil del docente competente de Humanidades y Ciencias Sociales en el bachillerato mexicano desde la perspectiva del alumno
}

The profile of the competent teacher of humanities and social sciences in a Mexican high school according to the point of view of the students

\author{
ERIKa López Rodríguez ${ }^{1}$ \\ elopez003@hotmail.com \\ Universidad Autónoma de Yucatán (UADY), México
}

\section{Resumen:}

En este trabajo se presenta el perfil que deben tener los docentes de Bachillerato en el área de Humanidades y Ciencias Sociales conforme las inquietudes expresadas por los estudiantes de una escuela pública vespertina del Poniente de Mérida. Se elaboró un instrumento en escala Likert con 48 ítems que midió doce competencias obtenidas de los trabajos de connotados investigadores y de la literatura oficial mexicana que sirvió como referente. El cuestionario empleado para esta investigación fue piloteado y validado por jueces, en su confiabilidad reportó 0.94 de alfa de Crombach.

Cada vez se torna necesario crear perfiles para puestos laborales, como es el caso de la docencia, a fin de crear parámetros observables de actuación profesional. En los

\begin{abstract}
:
This paper explains the profile that teachers in high school should have in the field of humanities and social sciences according to the aims expressed by the students of a public night high school in the West of Merida. Students were asked to complete a 48-item questionnaire designed on a Likert scale with a view to measuring twelve competences obtained from the work of prestigious researches and the official Mexican documents used as a referent. This questionnaire was validated by judges, had a pilot test and a Cronbach's alpha of 0.94. It is increasingly more necessary to create job profiles, including those for the teaching profession, with a view to establishing parameters for professional development. Those profiles should detail the dimensions which the job addresses and
\end{abstract}

1 Dirección para correspondencia (correspondence address):

Erika López Rodríguez. Universidad Autónoma de Yucatán (UADY), Mérida, Yucatán (México). 
El perfil del docente competente de Humanidades y Ciencias Sociales en el bachillerato mexicano desde la perspectiva del alumno

ERIKA López RODRíGUEZ

perfiles se detallan las dimensiones de actuación y los descriptores que dictaminan el modus operandi del quehacer áulico. En el modelo por competencias estos perfiles señalan las actitudes, habilidades y conocimientos que son deseables en un docente. Estos perfiles dan la pauta para hacer una adecuada evaluación de la docencia. Los resultados se analizaron a través del software estadístico SPSS v.21, mediante frecuencias y porcentajes y comparación de medias. En la interpretación se discriminó en favor de los polos positivos que implicaban la elección racional del alumno de la competencia docente. Se encontró que todas las competencias fueron importantes, mas no todos los descriptores resultaron del agrado de los alumnos. Al final se presenta un listado con las competencias mejor evaluadas: preparación académica, comunicación asertiva, liderazgo, evaluación y ecología educativa.

\section{Palabras clave:}

Competencia; docentes; perfil; bachillerato; ciencias sociales; humanidades. the descriptors which adequate teaching practice should include. In the education model by competences these profiles present the attitudes, abilities and knowledge that a teacher should have. These profiles also provide guidelines to undertake accurate teacher assessment. The results were analysed with the statistical software package SPSS v.21 and frequencies, percentages and comparison of means were obtained. The interpretation especially addressed students' positive opinions regarding what teaching competence should be. The study found that all the competences were important, but not all the descriptors satisfied the students' expectations. This study also shows a list with the most popular competences for teaching: academic background, assertive communication, leadership, evaluation and educational environment.

\section{Keywords:}

Competence; teachers; profile; high school; social sciences; humanities.

\section{Résumé:}

Dans ce travail, on présente le profil que doivent avoir enseignants des sciences humaines et sociales du secondaire à travers des préoccupations exprimées par les élèves d'une école publique de l'ouest de Merida. À cet effet, un instrument a été développé à l'échelle de Likert avec 48 ítems qui ont mesuré douze compétences issues des travaux des chercheurs de renom et de la littérature officielle mexicaine qui a servi de référence. Le questionnaire utilisé pour cette recherche a été mis à l'essai et validé par des juges, et il a rapporté la fiabilité alpha de Cronbach 0,94.

II est de plus en plus nécessaire de créer des profils pour le travail, comme dans l'enseignement, de créer des paramètres de performance observables. Ces profils détaiIlent le modus operandi qui doit être fait dans la salle de classe. Ces profils indiquent les attitudes, les compétences et connaissances attendues chez un enseignant. C'est grâce à ces profils éducatifs que l'enseignement peut être évalué correctement.

Les résultats ont été analysés en utilisant le logiciel statistique SPSS v.21, par le biais de fréquences et pourcentages et la moyenne de comparaison. Dans l'interprétation, on a utilisé les pôles positifs impliquant le choix rationnel de la compétence d'enseignement par l'élève. Il a été constaté que toutes les compétences sont importantes, mais que tous les descripteurs n'ont pas été appréciés par les élèves. La préparation académique, la communication assertive, le leadership, l'évaluation et l'écologie de l'éducation étaient les compétences les mieux évaluées.

\section{Mots clés :}

Compétence; enseignants; profil; école secondaire; sciences sociales; sciences humaines.

Fecha de recepción: 28-7-2016

Fecha de aceptación: 27-9-2017 


\section{Introducción}

Por sentido común se presume que los buenos maestros forman adecuadamente a sus alumnos en las dimensiones afectiva, moral, cognoscitiva. Pero señalar esto deja abierto el margen de interpretación, permitiendo a cada quien dar opiniones variopintas sobre el tema, las cuales pueden distraer la atención respecto a lo que se pretende obtener del trabajo docente e indicar qué aspectos se deben trabajar y cuáles son necesarios reforzar. La mística que antaño envolvía la función docente se ha ido perdiendo con el correr de los años debido a que ahora se torna imprescindible crear perfiles para cargos específicos que dictaminen la forma de trabajo a ejercer durante el tiempo de enseñanza y más aún durante el periodo de contratación. Esta situación común ya en el entorno empresarial es una realidad palpable en la escuela desde que se adoptó el modelo por competencias. Por esta razón y porque la definición de parámetros de actuación docente sirve como mecanismo de evaluación, se impone la necesidad de hacer una definición de los rasgos de los enseñantes para garantizar que ocupen una plaza docente los mejor capacitados.

En este trabajo se presenta el perfil del docente en Humanidades y Ciencias Sociales conforme a la valoración hecha por los alumnos de una escuela preparatoria vespertina pública de la ciudad de Mérida, en el afán de orientar los esfuerzos educativos desde una visión democratizadora que integra la voz del estudiante respecto a lo que se considera idóneo en el trabajo del docente.

En virtud de que los perfiles educativos son parte integral de la evaluación de la docencia al servir como parámetros de eficiencia y buenas prácticas educativas, empleados luego como mecanismos administrativos en la contratación y promoción de personal, para conocer la opinión de los alumnos se hizo una selección de características, dimensiones y atributos expuestos por la literatura especializada, que abarcan habilidades tanto sociales como actitudinales y de manejo del conocimiento en función de acercar los contextos de creación y generación de los saberes desde el modelo por competencias. 


\section{Marco teórico}

En el 2008 México implementó la Reforma Integral de la Educación Media Superior (RIEMS), que establece un nuevo modelo educativo basado en competencias. Educar en competencias implica crear situaciones de enseñanza que permitan a los alumnos tener posesión de la clase para que adquieran "el paquete de las competencias" (Cachón, 2009): saberes, habilidades, actitudes y valores. Este "paquete", entendiendo por ello a la tríada de conocimientos, actitudes y habilidades, tiene sustento teórico en las declaraciones que hizo Delors (1996) para la UNESCO respecto a los cuatro pilares fundamentales de la educación: aprender a ser, aprender a convivir (actitudes), aprender a conocer (saber) y aprender a hacer (habilidades).

La RIEMS (DOF, 2008) se articuló con base en los lineamientos establecidos en la Declaración de Bolonia, el Proyecto Tuning y el Alfa Tuning para América Latina (2004). De Bolonia recoge el Ilamamiento a las universidades para fomentar que la enseñanza de los alumnos no se limite al aula de suerte que se pudieran formar sociedades de conocimiento a través de la movilidad de los saberes, ya sea de forma física, como se pensó en un inicio o bien de forma virtual gracias al avance tecnológico. Esta recomendación favorece la consolidación del modelo por competencias al fomentar que los alumnos pongan en acción su conocimiento en contextos diversos, lo que a su vez favorece que los estudios adquiridos se vuelvan pertinentes en el ámbito global y continuamente se evalúen en función de su operatividad.

El Proyecto Tuning que primero se aplicó con universidades europeas y posteriormente fue emulado para América Latina, consolidó que la enseñanza se ejerciera bajo este nuevo modelo por competencias. Fue a partir del Proyecto Tuning que se introdujo en el escenario académico internacional la actual terminología que ahora está en casi todos los manuscritos educativos. Entre los términos recién adoptados está la palabra perfil, la cual se ha venido empleando para estructurar un criterio racional de idoneidad que sirva como parámetro para la evaluación de la docencia.

El primer perfil de la docencia surgió a partir del Proyecto Alfa Tuning para América Latina en el listado de las 27 competencias, mismas que fueron establecidas como un ideal de prácticas efectivas para aplicar en el aula. Este trabajo inspiró la creación del Acuerdo Secretarial 447 
de México el cual declara, en el marco de la RIEMS, que los docentes tienen que cubrir ciertas competencias para enseñar en bachillerato. Las competencias docentes requeridas en dicho documento son: 1. Organiza su formación continua a lo largo de su trayectoria profesional, 2. Domina y estructura los saberes para facilitar experiencias de aprendizaje significativo, 3. Planifica los procesos de enseñanza y aprendizaje y los ubica en contextos disciplinares, curriculares y sociales amplios, 4. Lleva a la práctica procesos de enseñanza y aprendizaje de manera efectiva, creativa e innovadora a su contexto institucional, 5. Evalúa los procesos de enseñanza y aprendizaje con un enfoque formativo, 6. Construye ambientes para el aprendizaje autónomo y colaborativo, 7 . Contribuye a la generación de un ambiente que facilite el desarrollo sano e integral de los estudiantes, 8. Participa en los proyectos de mejora continua de su escuela y apoya la gestión institucional (DOF, 2008).

Para 2013 la recién creada Ley General del Servicio Profesional Docente (LGSPD) reconoce como obligatoria la enseñanza del bachillerato en todo el país y asume como parte de sus funciones la definición y revisión de los perfiles docentes para establecer los parámetros de ingreso, egreso y promoción de docentes a todos los niveles educativos (DOF, 2013). Previo a esto la contratación de personal estaba en manos de los funcionarios públicos y administradores locales de los planteles escolares, en total opacidad respecto a sus políticas internas de adquisición de personal y aumento de horas de clase. Para solucionar este problema, el ingreso, promoción y destitución del cargo docente queda en manos del Instituto Nacional de la Evaluación de la Educación (INEE), que, aparte de las competencias citadas en el acuerdo secretarial, revisa si el docente cubre los perfiles señalados por este organismo evaluador y periódicamente propone otros con base en el modelo por competencias (2014, 2015a, 2015b).

Este entusiasmo por crear perfiles docentes ha hecho que se tengan dos clases de competencias: las propuestas por el INEE y las del Acuerdo 447 aún vigente; las cuales se distancian en tanto que para el órgano evaluador es más relevante lo cognoscitivo, mientras que el segundo parte de un referente genérico de habilidades didácticas. Esto complica el panorama del docente que labora durante cortos periodos de enseñanza enseñando materias que solamente se estudian por un solo semestre. Y cuando se les practica la evaluación docente, no hay forma de saber si posee el grado de idoneidad requerido debido a que en el terreno 
de la praxis lo que comúnmente sucede con los gestores educativos es que entregan horas de carga laboral a profesionistas con carreras afines a las asignaturas a impartir, dejando como irrelevante las habilidades de enseñanza o bien sin importar demasiado la pericia del docente en el manejo de los contenidos curriculares, como reportan los medios locales (Animal Político, 2015).

En las pasadas experiencias de evaluación docente las quejas de los administrativos apuntan a que los maestros no son idóneos. Hay casos donde la reprobación es muy elevada, del 51.5\% lo cual a juicio de Díaz-Barriga es señal del fallo del sistema educativo que permite el egreso de profesionales mal preparados o de gestores educativos incompetentes (UNAM, 2016).

En la evaluación del 2015, el INEE evaluó a todos los docentes de educación básica y media superior o bachillerato conforme al siguiente itinerario: 1) la emisión de un informe de cumplimiento de responsabilidades firmado por el director del plantel escolar, 2) la presentación de dos trabajos hechos por un alumno de alto rendimiento y otro por un alumno de bajo rendimiento académico más las evidencias del portafolio del docente para conformar el expediente de evidencias de enseñanza, 3) la presentación del examen de conocimientos y competencias laborales y 4) la elaboración de una planeación didáctica argumentada (Mancera, 2016).

A esta situación problemática se suma el entusiasmo de la academia por crear más que apuntan a constantes rediseños de perfiles docentes, demandando que los profesionales que enseñan por breves tiempo de horas-clase posean también otras capacidades que podrían caer en la responsabilidad de los administradores educativos. Para abundar en el tema conviene revisar las propuestas de las competencias docentes hechas por Perrenoud (en Fernández y Sánchez, 2014), Zabalza (2003), Cano (2008), Cornellas (2002), Scriven (en Rueda, 2009).

La palabra perfil es entendida como un conjunto de rasgos peculiares que caracterizan a alguien o algo (DRAE, 2015). En la LGSPD se define como perfil docente el conjunto de características, requisitos, cualidades o aptitudes que deberá tener el aspirante a desempeñar un puesto o función descrito específicamente (DOF, 2013). Debido a que los perfiles docentes nacieron dentro del modelo por competencias, pueden ser Ilamados como perfiles competenciales (Más, 2012). Para ser tales, estos perfiles que denotan competencias laborales para la enseñanza deben 
integrar descriptores de los conocimientos, habilidades y actitudes que se ponen en juego al momento de generar ambientes de aprendizaje.

En México la educación media superior (EMS), que corresponde al bachillerato, atiende a jóvenes cuyas edades están entre los 15 y los 21 años. Las áreas de estudio del bachillerato están comprendidas en cinco campos disciplinares: Humanidades, Comunicación, Ciencias Sociales, Ciencias Experimentales y Matemáticas. En este país el bachillerato es amplio y complejo, cada subsistema de enseñanza tiene su propia maIla curricular en virtud de los fines que se persiguen, que pueden ser la continuación del estudio en la universidad o entrar al campo laboral en calidad de técnico.

En varios subsistemas del nivel medio superior se les pide a los docentes que se reúnan con sus pares del campo disciplinar donde enseñan como parte habitual de las prácticas docentes; en la educación media superior se le denomina Academias. Pero a partir de la entrada en vigor del nuevo modelo educativo que inició en agosto de 2017, quedando aún por consolidar el anterior, el trabajo en colegiado es fundamental, pues pretende abatir la desigualdad en la enseñanza creando ambientes de enseñanza homogéneos que favorezcan la integración y equidad. Esto sin duda supone otra exigencia más respecto a las funciones que debe realizar el docente con la intención de homologar sus prácticas y estilo de conducción de la clase al de sus colegas.

En el caso del área de Humanidades y Ciencias Sociales que ocupan una buena parte de la malla curricular con las materias de Sociología, Derecho, Historia, Artes, Literatura, Filosofía, Economía, Administración, Docencia, etc. (INEE, 2015b) y que ahora se ampliarán con el nuevo modelo educativo, el cual también hace algunas ligeras modificaciones curriculares, como el caso de Literatura que será una especialidad terminal en los alumnos próximos al egreso brindando la oportunidad de su estudio durante más de un año y medio lectivo (SEP a, 2017; SEP b, 2017). Muchas veces ocurre que los docentes que imparten estas asignaturas son los mismos que se mantienen en el plantel sin importar los vientos de cambio que lanzan los rediseños curriculares y administrativos, dada su protección sindical o derecho de plaza vitalicia ganado en los recientes concursos para la promoción de horas. Esto significa que quien enseñan una asignatura del campo de Humanidades, también podría enseñar una de Sociales.

No obstante, pese a lo que señalan las noticias sobre la efervescencia 
magisterial en oposición a la evaluación docente, en México hay una amplia tradición de evaluar al maestro en función de los comentarios de los estudiantes, sobre todo en las escuelas privadas y en las universidades (Zambrano y cols., 2005). En contraparte, en el sector público se está comenzando a adquirir la cultura de la evaluación de forma periódica a partir del 2015 con premios y sanciones para quienes resulten reprobados, desde la verticalidad de la autoridad educativa, sin incluir la voz del alumnado, como sí ocurre en las escuelas particulares y universidades.

En las evaluaciones hechas por el alumno a sus formadores se pretende conocer la efectividad de los docentes. Esta situación ventajosa del alumno, pues es quien puede juzgar de viva voz lo hecho por su formador, es la razón por la cual desde la década de los noventas hasta el 2010 estuvo vigente el portal electrónico Rate my Professors para conocer el desempeño de más de tres mil profesores universitarios de Estados Unidos, Canadá e Inglaterra, cerrado por el natural encono que aún existe en el gremio magisterial sobre la evaluación hecha por el alumno, pues lo que comúnmente sucede es que el estudiante asume el rol de cliente, dejando de ser el producto a formar, lo que motiva a que los administradores educativos tomen decisiones dramáticas como el despido de maestros al término de cada ciclo escolar, favoreciendo la contratación de universitarios ávidos por tener un empleo aunque sea por pocas horas. Esto, en vez de mejorar las condiciones de enseñanza, las empeora porque impide el desarrollo de proyectos educativos de largo alcance, o bien, favorece la simulación del profesor que prefiere aprobar a todos sus alumnos a dar notas bajas por temor a perder el empleo (García, 2014).

Una postura intermedia que pretende mirar esta delicada situación desde una perspectiva holista para considerar todos los aspectos o dimensiones en los que se mueve el docente (Looney, 2011), aboga por incluir, con sus justas precauciones, la voz del estudiante. De hecho, es uno de los requisitos de la nueva docencia conocida como paradigma de educación centrado en el alumno (Más, 2012), el cual permite concientizar al estudiante respecto a los alcances y límites que pueden darse en el aula en la relación con sus docentes. Hacer esto crea un espíritu democrático para asumir y llevar a cabo los arreglos internos del salón de clase de forma responsable, pudiendo así dar cuenta de la efectividad del docente y el grado de compromiso del alumno con su propia formación.

En este nuevo paradigma de la enseñanza por competencias, el docente de Humanidades y Ciencias Sociales debe cubrir con un perfil 
acorde con los requerimientos áulicos que observa el estudiante en la interacción diaria, los cuales resultan más reales a los propuestos por la literatura o las administraciones de políticas educativas, pues algo que se ha visto desde la incorporación del modelo por competencias es que los docentes están saturados de actividades que bien podrían realizar otros agentes educativos. Hay muchas dizque funciones del docente que hasta podrían quitarse del ideario de buenas prácticas docentes, como Ilama Zabalza a los perfiles del profesorado (2003), como ejemplo de cómo los ideales se convierten en criterios de evaluación docente baste mencionar el del informe Alfa Tuning (2004), que recomienda que los docentes "analicen críticamente las políticas educativas" e "identifiquen y gestionen apoyos para atender las necesidades educativas específicas en diferentes contextos.

La conformación de un perfil docente que esté acorde con la realidad educativa de los alumnos en el bachillerato para el mega campo disciplinar de Humanidades y Ciencias Sociales bajo el modelo por competencias, tiene que reconocer ante todo que hay una amplia cantidad de profesionales que no tienen formación en Educación o que carecen de dominio de su materia de enseñanza. Así como ocurre con el paquete de las competencias, el debería cubrir con un paquete de dimensiones básicas de actuación áulica que fueron recogidas en este trabajo para conocer cuáles son las más valoradas por los alumnos.

\section{Marco empírico}

Para conocer las competencias docentes valoradas por los alumnos se procedió a la revisión de la literatura especializada, sobre todo en lo referente a las taxonomías de perfiles competenciales. Hecha la revisión se hicieron varias tablas comparativas para estimular el análisis heurístico, sobre todo por el contenido de los documentos a fin de extraer categorías afines entre los autores, esto se consiguió no sólo con los descriptores de las taxonomías sino con las explicaciones de los autores leídos (Piñuel, 2002). Así fue que se extrajeron doce dimensiones de actuación docente: liderazgo, organización, valores, preparación académica, alfabetización digital, habilidades docentes, evaluación, comunicación asertiva, integración social, identidad institucional, participación comunitaria y ecología educativa. 
Las categorías se usaron para diseñar un instrumento en escala Likert para medir las preferencias de los estudiantes en escala ordinal donde se asignó el valor de 1 a Nada Importante, 2 Poco Importante, 3 Importante y 4 Muy Importante. Cada categoría estuvo acompañada de cuatro descriptores, por lo que se tuvieron en total 48 ítems distribuidos en grupos de cuatro correspondientes a las doce dimensiones resultantes del análisis de los perfiles docentes para formar las competencias docentes deseadas por el alumnado.

Por el alcance de la investigación se trata de un estudio descriptivo aplicado a todo el universo de una escuela preparatoria pública vespertina del Poniente de Mérida en una zona urbana elegida por conveniencia para conocer qué competencias se valoran en el área de Humanidades y Ciencias Sociales.

En la portadilla del instrumento se incluyeron elementos tales como la presentación de la investigación, donde se describieron los alcances éticos y el derecho a no contestar de los alumnos. Para esto se tomó como referencia el manual APA en cuanto a cómo se debe estructurar el consentimiento informado. Y también se anexó una hoja de aspectos demográficos para realizar el análisis de las características de los sujetos, quienes respondieron de forma anónima. El censo se aplicó a cuatro grupos, tentativamente debieron participar 168 alumnos, pero en ese momento estaban sólo 109 estudiantes.

El instrumento fue piloteado en una preparatoria cercana con una muestra elegida al azar de 21 estudiantes que comparte las mismas características con la población objeto de estudio. La confiabilidad del instrumento reportó un Alfa de Crombach de 0.94, considerado por George y Mallery (2003, p. 231) como excelente. La validez se obtuvo por jueces expertos en el tema que son catedráticos de la Universidad Autónoma de Yucatán y la Universidad Autónoma de Tlaxcala. Ya en la aplicación del censo, el cual fue administrado de forma impresa, se le pidió a los alumnos que sólo evaluaran a sus docentes del área objeto de estudio.

\section{Resultados y discusión}

Los resultados del censo arrojaron que las doce competencias docentes halladas en la literatura especializada son importantes para los alumnos pues todas fueron consideradas como Importantes y Muy Importantes en 
un alto porcentaje. Para el análisis y discusión se procedió a realizar una comparación de medias y por t Student entre géneros y grupos a fin de discriminar las preferencias particulares. En vista de que no se obtuvo un porcentaje mayoritario en las respuestas negativas, se hizo una lista de prelación con la comparación de medias. Para todo lo anterior se usó el software estadístico SPSS v.21 que sirvió de mucho en la interpretación de datos.

En más de un 90\% los alumnos se decantaron por tener docentes que sean líderes dentro del aula, con capacidad de motivación, dispuestos a captar la atención de sus alumnos, ponerlos a trabajar en equipo y con espíritu de servicio para atender sus inquietudes. El 68.8\% cree que es muy importante que sus maestros se esfuercen en captar su atención dentro del aula. Para el $66.1 \%$ de la población los maestros deben motivar sin emplear las ofensas o sarcasmos. Pocos alumnos opinan que la conformación de grupos cooperativos en el aula es algo que deba realizar el docente (Tabla 1).

Tabla 1. Competencia Liderazgo.

\begin{tabular}{|c|c|c|c|c|c|c|c|c|}
\hline \multirow[t]{2}{*}{ Aspecto estudiado } & \multicolumn{2}{|c|}{$\begin{array}{c}\text { Nada } \\
\text { importante }\end{array}$} & \multicolumn{2}{|c|}{$\begin{array}{c}\text { Poco } \\
\text { importante }\end{array}$} & \multicolumn{2}{|c|}{ Importante } & \multicolumn{2}{|c|}{$\begin{array}{c}\text { Muy } \\
\text { importante }\end{array}$} \\
\hline & $f$ & $\%$ & $f$ & $\%$ & $f$ & $\%$ & $f$ & $\%$ \\
\hline $\begin{array}{l}\text { Los maestros deben ser } \\
\text { capaces de captar la aten- } \\
\text { ción de sus estudiantes. }\end{array}$ & 0 & 0 & 5 & 4.6 & 29 & 26.6 & 75 & 68.8 \\
\hline $\begin{array}{l}\text { Los maestros deben fo- } \\
\text { mentar la cooperación en } \\
\text { vez de la competencia en- } \\
\text { tre alumnos. }\end{array}$ & 0 & 0 & 14 & 12.8 & 47 & 43.1 & 47 & 43.1 \\
\hline $\begin{array}{l}\text { Los maestros deben tener } \\
\text { actitud de servicio como } \\
\text { disponibilidad de escu- } \\
\text { cha y la atención a las ne- } \\
\text { cesidades del aula. }\end{array}$ & 3 & 2.8 & 10 & 9.2 & 31 & 28.4 & 65 & 59.6 \\
\hline $\begin{array}{l}\text { Los maestros deben ha- } \\
\text { cernos sentir que somos } \\
\text { capaces de alcanzar me- } \\
\text { tas elevadas sin tener que } \\
\text { ofendernos. }\end{array}$ & 1 & .9 & 9 & 8.3 & 25 & 22.9 & 72 & 66.1 \\
\hline
\end{tabular}

El 62.2\% opina que los maestros deben ser capaces de diseñar actividades de aprendizaje que les permitan acceder a los conocimientos de 
la asignatura. Para el $49.5 \%$ las actividades de aprendizaje deben seguir un orden gradual, siguiendo secuencias didácticas que permitan acceder a mayores niveles de complejidad. En menor proporción de importancia, con un $38.5 \%$, están quienes creen que sus docentes deban diseñar tareas que impliquen la participación grupal en el aula (Tabla 2).

Tabla 2. Competencia Organización.

\begin{tabular}{|c|c|c|c|c|c|c|c|c|}
\hline \multirow[t]{2}{*}{ Aspecto estudiado } & \multicolumn{2}{|c|}{$\begin{array}{c}\text { Nada } \\
\text { importante }\end{array}$} & \multicolumn{2}{|c|}{$\begin{array}{c}\text { Poco } \\
\text { importante }\end{array}$} & \multicolumn{2}{|c|}{ Importante } & \multicolumn{2}{|c|}{$\begin{array}{c}\text { Muy } \\
\text { importante }\end{array}$} \\
\hline & $f$ & $\%$ & $f$ & $\%$ & $f$ & $\%$ & $\mathrm{f}$ & $\%$ \\
\hline $\begin{array}{l}\text { Los maestros deben ser } \\
\text { capaces de diseñar activi- } \\
\text { dades de aprendizaje que } \\
\text { permitan que sus alumnos } \\
\text { se apropien de los cono- } \\
\text { cimientos de la asignatura. }\end{array}$ & 0 & 0 & 5 & 4.6 & 34 & 31.2 & 68 & 62.4 \\
\hline $\begin{array}{l}\text { Los profesores deben par- } \\
\text { tir de conocimientos sim- } \\
\text { ples e ir avanzando hacia } \\
\text { temas más complejos. }\end{array}$ & 0 & 0 & 8 & 7.3 & 47 & 43.1 & 54 & 49.5 \\
\hline $\begin{array}{l}\text { Los maestros deben ser } \\
\text { capaces de diseñar activi- } \\
\text { dades de aprendizaje que } \\
\text { recojan todos los conteni- } \\
\text { dos vistos en clase }\end{array}$ & 2 & 1.8 & 11 & 10.1 & 46 & 42.2 & 49 & 45 \\
\hline $\begin{array}{l}\text { Los profesores deben } \\
\text { promover que todos sus } \\
\text { alumnos participen en las } \\
\text { clases. }\end{array}$ & 2 & 1.8 & 16 & 14.7 & 48 & 44 & 42 & 38.5 \\
\hline
\end{tabular}

Respecto a los valores, misma que hace referencia a los asuntos éticos, los alumnos opinaron en un $20 \%$ que están en desacuerdo con que sus maestros les Ilamen la atención fuera del salón de clase. Por lo contrario, aprueban que sus docentes sean ejemplos a seguir por su rectitud de vida dentro y fuera del aula (Figura 1). 
El perfil del docente competente de Humanidades y Ciencias Sociales en el bachillerato mexicano desde la perspectiva del alumno

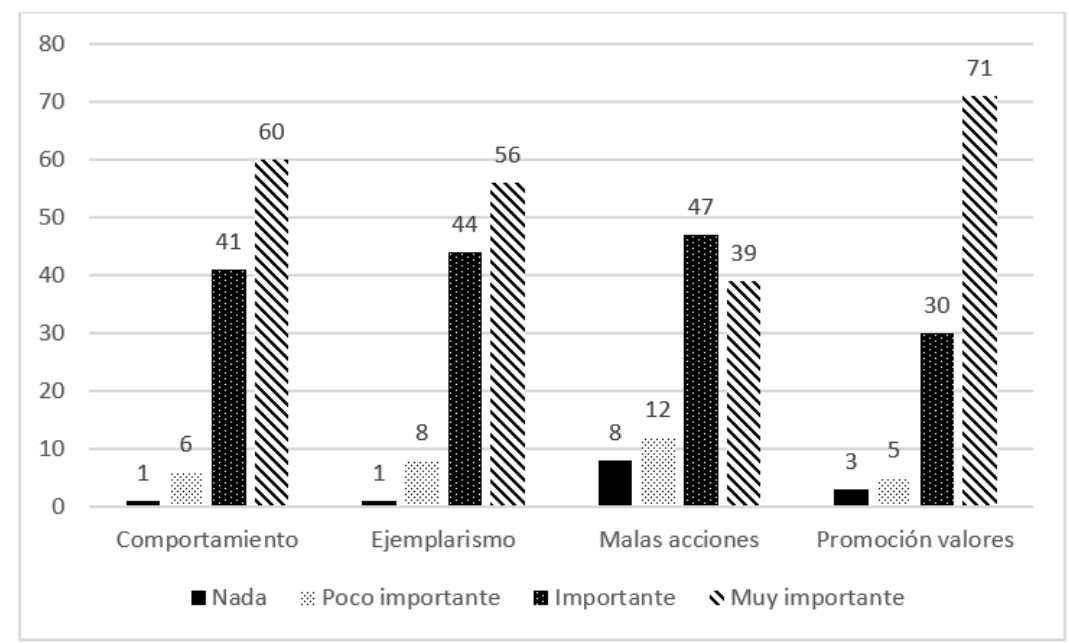

Figura 1. Análisis de la importancia de los valores en el profesor.

Un $80.7 \%$ de los estudiantes consideró que sus docentes están obligados a dominar sus contenidos de estudio. El 62.4\% se mostró a favor de la actualización constante, lo que supone inversión de horas de estudio para el profesor. El 49.5\% valoró importante que los docentes conozcan más su contenido de enseñanza y en un $57.8 \%$ que sean profesionistas afines a su materia de enseñanza (Tabla 3).

Tabla 3. Competencia Preparación académica.

\begin{tabular}{|c|c|c|c|c|c|c|c|c|}
\hline \multirow[t]{2}{*}{ Aspecto estudiado } & \multicolumn{2}{|c|}{$\begin{array}{c}\text { Nada } \\
\text { importante }\end{array}$} & \multicolumn{2}{|c|}{$\begin{array}{c}\text { Poco } \\
\text { importante }\end{array}$} & \multicolumn{2}{|c|}{ Importante } & \multicolumn{2}{|c|}{$\begin{array}{c}\text { Muy } \\
\text { importante }\end{array}$} \\
\hline & f & $\%$ & $\mathrm{f}$ & $\%$ & $f$ & $\%$ & f & $\%$ \\
\hline $\begin{array}{l}\text { Es importante que los } \\
\text { maestros dominen los te- } \\
\text { mas que enseñan. }\end{array}$ & 2 & 1.8 & 1 & .9 & 18 & 16.5 & 88 & 80.7 \\
\hline $\begin{array}{l}\text { Los profesores deben de te- } \\
\text { ner una formación afín con } \\
\text { los temas que enseñan. }\end{array}$ & 0 & 0 & 5 & 4.6 & 41 & 37.6 & 63 & 57.8 \\
\hline $\begin{array}{l}\text { Los profesores deben pro- } \\
\text { curar estar actualizados } \\
\text { en los contenidos de las } \\
\text { asignaturas que imparten. }\end{array}$ & 1 & .9 & 5 & 4.6 & 35 & 32.1 & 68 & 62.4 \\
\hline $\begin{array}{l}\text { Los maestros deben se- } \\
\text { guir estudiando cuestio- } \\
\text { nes vinculadas con su } \\
\text { propia formación }\end{array}$ & 3 & 2.8 & 9 & 8.3 & 42 & 38.5 & 54 & 49.5 \\
\hline
\end{tabular}


El perfil del docente competente de Humanidades y Ciencias Sociales en el bachillerato mexicano desde la perspectiva del alumno

En relación a la alfabetización digital los alumnos valoraron por arriba del $70 \%$ las habilidades digitales del maestro y que éste considere el empleo de recursos informáticos para el aula. Sin embargo, las respuestas varían en relación con la ampliación del canal comunicativo maestro-alumno como complemento de la clase (Figura 2).

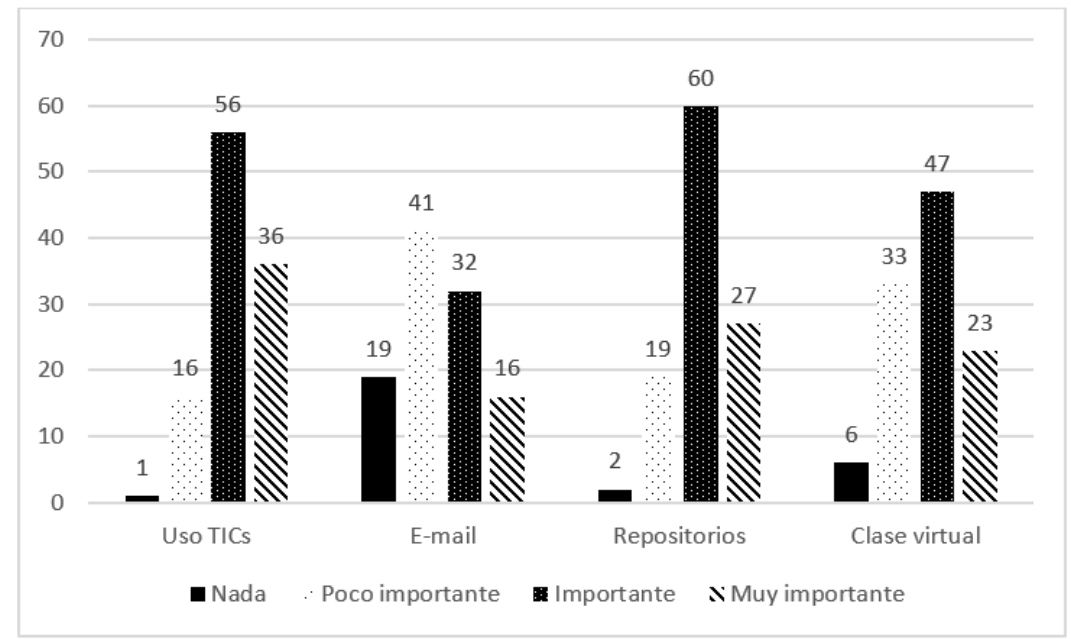

Figura 2. Competencia Alfabetización Digital.

Sobre las habilidades docentes los alumnos consideraron en más de un $65 \%$ que no es muy importante que sus docentes realicen sus materiales didácticos. Pero sí es relevante que los docentes ayuden a sus estudiantes a conseguir sus metas y ser creativos (Tabla 4).

Tabla 4. Competencia Habilidades docentes.

\begin{tabular}{|c|c|c|c|c|c|c|c|c|}
\hline \multirow[t]{2}{*}{ Aspecto estudiado } & \multicolumn{2}{|c|}{$\begin{array}{c}\text { Nada } \\
\text { importante }\end{array}$} & \multicolumn{2}{|c|}{$\begin{array}{c}\text { Poco } \\
\text { importante }\end{array}$} & \multicolumn{2}{|c|}{ Importante } & \multicolumn{2}{|c|}{$\begin{array}{c}\text { Muy } \\
\text { importante }\end{array}$} \\
\hline & $f$ & $\%$ & f & $\%$ & f & $\%$ & f & $\%$ \\
\hline $\begin{array}{l}\text { Los profesores deben crear } \\
\text { sus materiales didácticos. }\end{array}$ & 4 & 3.7 & 18 & 16.5 & 50 & 45.9 & 37 & 33.9 \\
\hline $\begin{array}{l}\text { Los profesores deben ser } \\
\text { cercanos a los estudiantes } \\
\text { para brindarles orientación. }\end{array}$ & 1 & .9 & 15 & 13.8 & 56 & 51.4 & 37 & 33.9 \\
\hline $\begin{array}{l}\text { Las estrategias de los } \\
\text { maestros deben tener me- } \\
\text { tas que ayuden a alcanzar } \\
\text { las competencias. }\end{array}$ & 3 & 2.8 & 8 & 7.3 & 46 & 42.2 & 52 & 47.7 \\
\hline $\begin{array}{l}\text { Las actividades deben de- } \\
\text { sarrollar la creatividad. }\end{array}$ & 0 & 0 & 3 & 2.8 & 53 & 48.6 & 53 & 48.6 \\
\hline
\end{tabular}


En total acuerdo se mostraron respecto a la transparencia de resultados, que los docentes empleen rúbricas para evaluar y diferentes formas de evaluación para comprender todas las dimensiones en las que actúa el alumno. Sin embargo, hay un encono en cuanto que sea integradas las actitudes y valores para conseguir una evaluación integral (Figura 3).

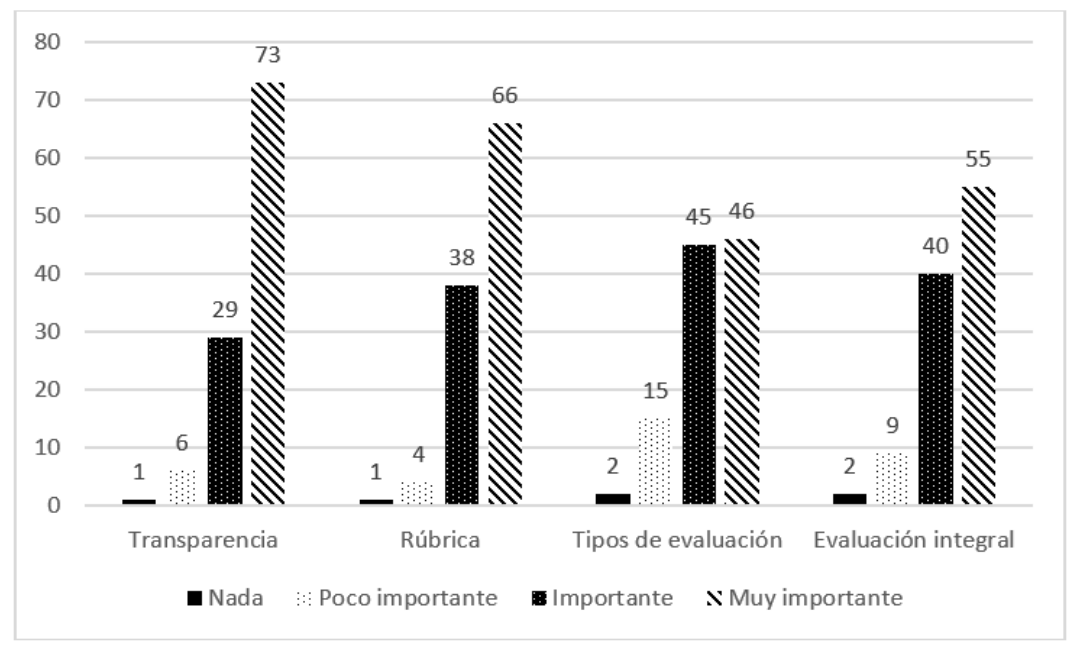

Figura 3. Competencia Evaluación.

En un $67 \%$ el alumnado discrepa respecto al involucramiento de los padres de familia en los asuntos académicos de los adolescentes. Por lo demás, se muestran favorables porque las exposiciones sean claras, que los docentes sean congruentes y que integren las opiniones y dudas en su exposición (Tabla 5).

Tabla 5. Competencia Comunicación asertiva.

\begin{tabular}{|c|c|c|c|c|c|c|c|c|}
\hline \multirow[t]{2}{*}{ Aspecto estudiado } & \multicolumn{2}{|c|}{$\begin{array}{c}\text { Nada } \\
\text { importante }\end{array}$} & \multicolumn{2}{|c|}{$\begin{array}{c}\text { Poco } \\
\text { importante }\end{array}$} & \multicolumn{2}{|c|}{ Importante } & \multicolumn{2}{|c|}{$\begin{array}{c}\text { Muy } \\
\text { importante }\end{array}$} \\
\hline & $\mathrm{f}$ & $\%$ & $\mathrm{f}$ & $\%$ & $f$ & $\%$ & f & $\%$ \\
\hline $\begin{array}{l}\text { Los maestros deben de } \\
\text { explicar con claridad los } \\
\text { temas. }\end{array}$ & 2 & 1.8 & 2 & 1.8 & 13 & 11.9 & 92 & 84.4 \\
\hline $\begin{array}{l}\text { Los maestros deben de ser } \\
\text { congruentes en su comu- } \\
\text { nicación verbal y no ver- } \\
\text { bal. }\end{array}$ & 1 & .9 & 4 & 3.7 & 31 & 28.4 & 73 & 67 \\
\hline
\end{tabular}


El perfil del docente competente de Humanidades y Ciencias Sociales en el bachillerato mexicano desde la perspectiva del alumno

ERIKA LÓPEZ RODRíGUEZ

\begin{tabular}{|c|c|c|c|c|c|c|c|c|}
\hline \multirow[t]{2}{*}{ Aspecto estudiado } & \multicolumn{2}{|c|}{$\begin{array}{c}\text { Nada } \\
\text { importante }\end{array}$} & \multicolumn{2}{|c|}{$\begin{array}{c}\text { Poco } \\
\text { importante }\end{array}$} & \multicolumn{2}{|c|}{ Importante } & \multicolumn{2}{|c|}{$\begin{array}{c}\text { Muy } \\
\text { importante }\end{array}$} \\
\hline & $f$ & $\%$ & $f$ & $\%$ & $f$ & $\%$ & f & $\%$ \\
\hline $\begin{array}{l}\text { Los maestros deben de } \\
\text { incorporar las dudas del } \\
\text { grupo a sus explicaciones. }\end{array}$ & 1 & .9 & 2 & 1.8 & 24 & 22 & 81 & 74.3 \\
\hline $\begin{array}{l}\text { Los maestros deben plati- } \\
\text { car con mis padres o tuto- } \\
\text { res para que tenga apoyo } \\
\text { moral en casa. }\end{array}$ & 10 & 9.2 & 26 & 23.9 & 37 & 33.9 & 36 & 33 \\
\hline
\end{tabular}

De forma general se puede señalar que para los alumnos es importante que sus docentes estén satisfechos de trabajar en la escuela donde están y que reflejen a su institución, pero también que los planteles reconozcan a sus mejores elementos y los asignen a las aulas donde pueden tener un buen desempeño (Figura 4).

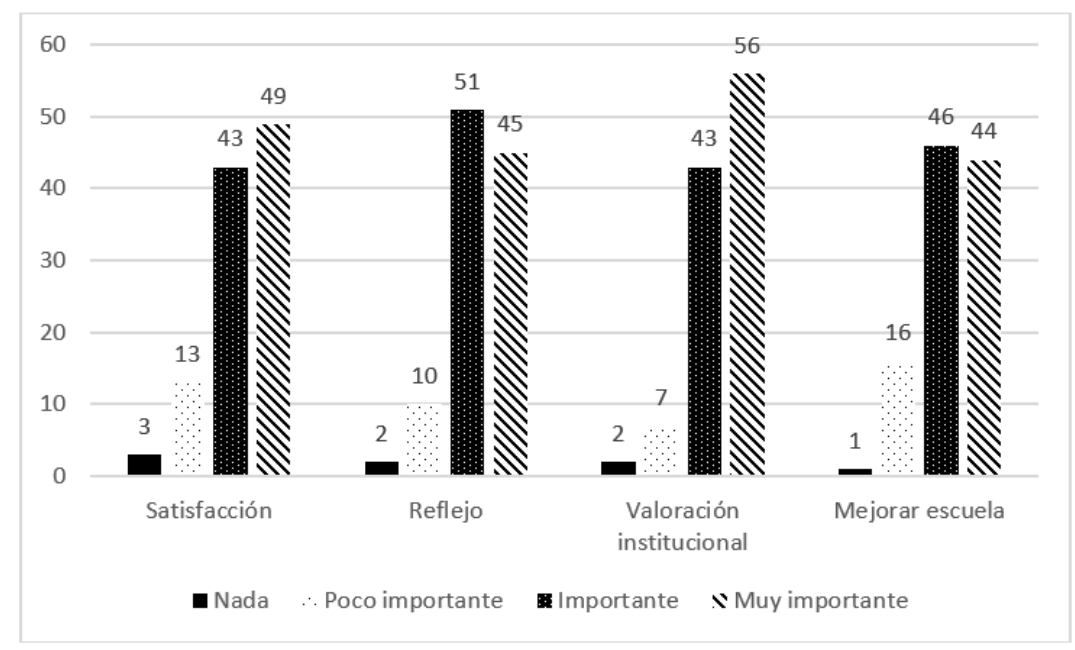

Figura 4. Competencia Identidad institucional.

En un $35.8 \%$ los alumnos prefieren que sus docentes no los involucren en las actividades que realiza el colegio, ni que los pongan a trabajar en equipos interdisciplinares donde se ven obligados a colaborar con gente que les resulta poco familiar (35.8\%). Sin embargo, es importante que el docente promueva estas actividades culturales y deportivas y de trabajo en equipo (Tabla 6). 
Tabla 6. Competencia Integración social.

\begin{tabular}{|c|c|c|c|c|c|c|c|c|}
\hline \multirow[t]{2}{*}{ Aspecto estudiado } & \multicolumn{2}{|c|}{$\begin{array}{c}\text { Nada } \\
\text { importante }\end{array}$} & \multicolumn{2}{|c|}{$\begin{array}{c}\text { Poco } \\
\text { importante }\end{array}$} & \multicolumn{2}{|c|}{ Importante } & \multicolumn{2}{|c|}{$\begin{array}{c}\text { Muy } \\
\text { importante }\end{array}$} \\
\hline & $f$ & $\%$ & $f$ & $\%$ & f & $\%$ & $f$ & $\%$ \\
\hline $\begin{array}{l}\text { Los maestros deben invo- } \\
\text { lucrar a sus alumnos en los } \\
\text { proyectos de la escuela. }\end{array}$ & 3 & 2.8 & 14 & 12.8 & 52 & 47.7 & 39 & 35.8 \\
\hline $\begin{array}{l}\text { Los maestros deben mos- } \\
\text { trar interés y ponerse la } \\
\text { camiseta participando en } \\
\text { las actividades que reali- } \\
\text { za la escuela. }\end{array}$ & 2 & 1.8 & 19 & 17.4 & 43 & 39.4 & 44 & 40.4 \\
\hline $\begin{array}{l}\text { Los maestros deben traba- } \\
\text { jar cooperativamente en } \\
\text { proyectos interdisciplinares. }\end{array}$ & 3 & 2.8 & 14 & 12.8 & 52 & 47.7 & 39 & 35.8 \\
\hline $\begin{array}{l}\text { Los profesores deben de } \\
\text { promover el trabajo en } \\
\text { equipos. }\end{array}$ & 5 & 4.6 & 10 & 9.2 & 43 & 39.4 & 49 & 45 \\
\hline
\end{tabular}

Es relevante que los maestros hagan actividades extraescolares en las que participen los alumnos, que los motiven a ser agentes de cambio social, ser solidarios con el prójimo y que promueva el pensamiento crítico (Tabla 7).

Tabla 7. Competencia Participación comunitaria.

\begin{tabular}{|c|c|c|c|c|c|c|c|c|}
\hline \multirow[t]{2}{*}{ Aspecto estudiado } & \multicolumn{2}{|c|}{$\begin{array}{c}\text { Nada } \\
\text { importante }\end{array}$} & \multicolumn{2}{|c|}{$\begin{array}{c}\text { Poco } \\
\text { importante }\end{array}$} & \multicolumn{2}{|c|}{ Importante } & \multicolumn{2}{|c|}{$\begin{array}{c}\text { Muy } \\
\text { importante }\end{array}$} \\
\hline & $\mathrm{f}$ & $\%$ & $f$ & $\%$ & $\mathrm{f}$ & $\%$ & $f$ & $\%$ \\
\hline $\begin{array}{l}\text { Los maestros deben parti- } \\
\text { cipar con sus alumnos en } \\
\text { actividades promovidas } \\
\text { por el gobierno. }\end{array}$ & 7 & 6.4 & 27 & 24.8 & 44 & 40.4 & 30 & 27.5 \\
\hline $\begin{array}{l}\text { Los maestros deben promo- } \\
\text { ver que sus alumnos sean } \\
\text { agentes de cambio social. }\end{array}$ & 2 & 1.8 & 18 & 16.5 & 52 & 47.7 & 36 & 33 \\
\hline $\begin{array}{l}\text { Los maestros deben fo- } \\
\text { mentar la solidaridad con } \\
\text { el prójimo dentro y fuera } \\
\text { de la escuela. }\end{array}$ & 4 & 3.7 & 16 & 14.7 & 42 & 38.5 & 46 & 42.2 \\
\hline $\begin{array}{l}\text { Los maestros deben hacer- } \\
\text { me reflexionar críticamen- } \\
\text { te acerca de la situación } \\
\text { que vivimos en México. }\end{array}$ & 6 & 5.5 & 14 & 12.8 & 37 & 33.9 & 50 & 45.9 \\
\hline
\end{tabular}


El perfil del docente competente de Humanidades y Ciencias Sociales en el bachillerato mexicano desde la perspectiva del alumno

La competencia nombrada como ecología educativa hace referencia al ambiente de clase y sus elementos circundantes. En ella los alumnos valoraron positivamente que sus docentes creen un clima de óptimo de aprendizaje, que debe ser extensivo también con los colegas docentes. Esto implica sobremanera el control del ruido externo que en las áreas urbanas tiende a ser un reto por la contaminación sonora de la calle (Tabla 8).

Tabla 8. Competencia Ecología educativa.

\begin{tabular}{|c|c|c|c|c|c|c|c|c|}
\hline \multirow[t]{2}{*}{ Aspecto estudiado } & \multicolumn{2}{|c|}{$\begin{array}{c}\text { Nada } \\
\text { importante }\end{array}$} & \multicolumn{2}{|c|}{$\begin{array}{c}\text { Poco } \\
\text { importante }\end{array}$} & \multicolumn{2}{|c|}{ Importante } & \multicolumn{2}{|c|}{$\begin{array}{c}\text { Muy } \\
\text { importante }\end{array}$} \\
\hline & f & $\%$ & $f$ & $\%$ & f & $\%$ & f & $\%$ \\
\hline $\begin{array}{l}\text { Los maestros deben de } \\
\text { crear un ambiente agra- } \\
\text { dable en el aula que me } \\
\text { permita aprender. }\end{array}$ & 3 & 2.8 & 4 & 3.7 & 33 & 30.3 & 68 & 62.4 \\
\hline $\begin{array}{l}\text { Los maestros deben de } \\
\text { eliminar las distracciones } \\
\text { durante su clase, sean és- } \\
\text { tos personas o situaciones } \\
\text { que impidan la compren- } \\
\text { sión de sus temas. }\end{array}$ & 4 & 3.7 & 7 & 6.4 & 34 & 31.2 & 62 & 56.9 \\
\hline $\begin{array}{l}\text { Los maestros deben fo- } \\
\text { mentar un sano clima de } \\
\text { enseñanza en el aula an- } \\
\text { tes de dar su clase. }\end{array}$ & 0 & 0 & 14 & 12.8 & 44 & 40.4 & 50 & 45.9 \\
\hline $\begin{array}{l}\text { Los maestros deben cui- } \\
\text { dar que su entorno con } \\
\text { sus colegas sea agradable } \\
\text { para que los alumnos se } \\
\text { sientan motivados a in- } \\
\text { teractuar con todos los } \\
\text { docentes, sin temor a re- } \\
\text { presalias. }\end{array}$ & 2 & 1.8 & 6 & 5.5 & 36 & 33 & 64 & 58.7 \\
\hline
\end{tabular}

Las competencias más valoradas fueron: 1. Preparación académica, 2. Comunicación asertiva, 3. Liderazgo, 4. Evaluación y 5. Ecología educativa (Tabla 9). 
El perfil del docente competente de Humanidades y Ciencias Sociales en el bachillerato mexicano desde la perspectiva del alumno

Tabla 9. Comparación de medias de las competencias valoradas por los alumnos.

\begin{tabular}{lcc}
\hline Competencias & N & Media \\
\hline Liderazgo & 106 & 13.94 \\
Organización & 106 & 13.53 \\
Valores & 105 & 13.61 \\
Preparación académica & 108 & 14.25 \\
Alfabetización digital & 107 & 11.42 \\
Habilidades docentes & 109 & 13.09 \\
Evaluación & 106 & 13.84 \\
Comunicación asertiva & 108 & 14.03 \\
Integración social & 107 & 12.78 \\
Identidad institucional & 107 & 13.22 \\
Participación comunitaria & 107 & 12.49 \\
Ecología educativa & 107 & 13.80 \\
\hline
\end{tabular}

De las comparaciones entre grupos se apreció que para el grupo B casi todas las competencias resultaron pertinentes, no así para el grupo A. Esta variación refleja que no todos los grupos aprecian por igual los esfuerzos de sus formadores, lo que evidencia el carácter de la personalidad colectiva propia de los grupos (Tabla 10).

Tabla 10. Comparación de los porcentajes de aprobación de cada competencia.

\begin{tabular}{lcccc}
\hline Competencias & \multicolumn{4}{c}{ Grupos } \\
\hline & A & B & C & D \\
Valores & 18 & 25 & 20 & 20 \\
Preparación académica & 18 & 26 & 21 & 21 \\
Alfabetización digital & 18 & 26 & 20 & 22 \\
Habilidades docentes & 18 & 26 & 21 & 22 \\
Evaluación & 18 & 26 & 21 & 21 \\
Comunicación asertiva & 18 & 26 & 20 & 22 \\
\hline
\end{tabular}

Por último, se apreciaron diferencias significativas entre hombres y mujeres. Para los varones, pese a que fueron pocos en comparación con las mujeres, la evaluación docente fue más severa en casi todas las competencias al dar bajas puntuaciones. 
El perfil del docente competente de Humanidades y Ciencias Sociales en el bachillerato mexicano desde la perspectiva del alumno

ERIKA LÓPEZ RODRÍGUEZ

\begin{tabular}{cccccc}
\hline Competencia & Género & $\mathbf{N}$ & Media & $\mathbf{t}$ & Sig. (bilateral) \\
\hline \multirow{2}{*}{ Liderazgo } & Masculino & 33 & 13.30 & -2.266 & .026 \\
\cline { 2 - 6 } & Femenino & 73 & 14.23 & -2.100 & .041 \\
\hline \multirow{2}{*}{ Comunicación Asertiva } & Masculino & 33 & 13.45 & -2.152 & .034 \\
\cline { 2 - 6 } & Femenino & 75 & 14.28 & -1.807 & .078 \\
\hline Participación Comunitaria & Masculino & 33 & 11.73 & -2.067 & .041 \\
\cline { 2 - 6 } & Femenino & 74 & 12.82 & -1.938 & .058 \\
\hline Ecología educativa & Masculino & 33 & 13.03 & -2.477 & .015 \\
\cline { 2 - 5 } & Femenino & 74 & 14.15 & -2.477 & .016 \\
\hline
\end{tabular}

Hay coyunturas de estos resultados con la literatura en los tópicos que resultaron de interés para los estudiantes. De Zabalza (2003) se valida el hecho de captar la atención del aula, motivar a los estudiantes a permanecer durante la sesión de clase hasta el término del ciclo escolar, planificar el proceso de enseñanza-aprendizaje, dominar los temas que se enseñan, hacer transparente la evaluación, ser congruente en la comunicación oral, escrita y actitudinal; pero no respecto a la creación de materiales de enseñanza por parte de los maestros.

De Perrenoud (en Fernández y Sánchez, 2014) se acepta que los docentes sean promotores de una vida ética siendo ellos mismos sujetos ejemplares, pero esto siempre que no se involucre a los propios padres de familia en asuntos de disciplina. Para los alumnos la formación moral es un asunto académico y no de formación familiar.

En contraste con este mismo autor referido, los alumnos no están convencidos acerca del empleo de los recursos informáticos para extender el poder del docente hasta la ubicuidad del ciberespacio, ni que la clase se invierta dejando todo para la casa y casi nada para la escuela.

Por último, se validaron los documentos oficiales al considerar importantes la actualización docente y el dominio de temas (DOF, 2013), así como el mejoramiento del clima de aprendizaje para el que se necesita la sana cooperación entre pares y la evaluación de la función docente (DOF, 2008).

\section{Conclusión}

Las competencias expuestas por la RIEMS y el INEE, aparte de las demás empleadas para la elaboración del instrumento aplicado a los alumnos 
de una escuela vespertina de bachillerato en la ciudad de Mérida, son importantes y deberían ser consideradas para la elaboración de futuros instrumentos que permitan conocer la calidad de la docencia. Los resultados de los análisis por frecuencia y porcentaje dieron a conocer que para los alumnos resulta importante que sus docentes del área de Humanidades y Ciencias Sociales posean las doce competencias que figuraron en este trabajo como dimensiones de la enseñanza.

Es vital que los docentes tengan una sólida preparación académica, que puedan comunicarse con asertividad en cualquier contexto donde se les requiera, que tengan liderazgo dentro del aula y hasta fuera del mismo para mantener motivados a los alumnos, que empleen adecuadas estrategias para hacer la evaluación y sean capaces de crear entornos agradables de enseñanza.

Con esto se pudo crear el perfil genérico del docente atendiendo de forma puntual los descriptores de cada dimensión de enseñanza, por lo que queda como sigue.

El docente de educación media superior del área de Humanidades y Ciencias Sociales debe poseer un amplio conocimiento de sus temas de estudio para poder aclarar dudas en el aula y señalar los temas punteros que se están desarrollando actualmente, debido a que es una persona con gusto y motivación para aprender por cuenta propia a lo largo de su trayecto profesional.

Este docente debe saber comunicarse con propiedad en distintos medios y escenarios sociales con cualquier interlocutor; ser congruente de su mensaje emitido tanto de modo verbal como por medio de sus señas físicas.

Es un líder que motiva a sus alumnos, capta la atención de su grupo, promueve la creación de redes de cooperación entre los estudiantes y organiza el aula.

Evalúa conforme a varios criterios, promoviendo que haya más de una fuente para la obtención de las evidencias de los logros académicos realizados por los estudiantes, los cuales califica empleando rúbricas o listas de cotejo para hacer transparente el proceso para asignar calificaciones en las dimensiones ser, convivir, hacer y saber; haciendo así una evaluación holística del proceso de enseñanza-aprendizaje.

Por último, crea sanos ambientes de enseñanza-aprendizaje para favorecer el crecimiento del educando, por lo que elimina cualquier tipo de distracción y promueve la cordialidad con sus pares. 
El perfil del docente competente de Humanidades y Ciencias Sociales en el bachillerato mexicano desde la perspectiva del alumno

ERIKA LÓPEZ RODRÍGUEZ

\section{Referencias}

Cachón, C.; Mena, E. (2009) Modelo de Competencias para la Evaluación del Desempeño de Docentes de Escuelas Normales de Educación Primaria. X Congreso Nacional de Investigación Educativa. Recuperado de http://www.comie.org.mx /congreso/memoriaelectronica/v10/pdf/area_tematica_15/ponencias/13 23-F.pdf

Cano, M. (2008). La evaluación por competencias en la educación superior. Revista de Currículum y Formación del Profesorado, 12 (3). Recuperado de http://www.ugr. es/ recfpro/rev123COL1.pdf

Cornellas, M. (Comp.). (2002). Las competencias del profesorado para la acción tutorial. Barcelona: Praxis.

Delors, J. (1996). La educación encierra un tesoro. Informe a la UNESCO de la Comisión Internacional sobre la Educación para el siglo XXI. Madrid: Santillana.

DOF (2008). Acuerdo Secretarial 447. Recuperado de http://www.cbachilleres. edu. mex/cb/comunidad/docentes/pdf/Reforma_curricular/Acuerdos/ACUERDOS_RIEM/ ACUERDO447. pdf

DOF (2013). Ley General del Servicio Profesional Docente. Recuperado de http://www. dof.gob.mx/nota_detalle.php?codigo $=5313843 \&$ fecha $=11 / 09 / 2013$

Fernández, R.; Sánchez, L. (2014) España: Competencias docentes en secundaria. Análisis de perfiles de profesorado. Relieve. Vol. 20 (1).

García, J. (2014) ¿Para qué sirve la evaluación de la docencia? Un estudio exploratorio de las creencias de los estudiantes universitarios. Archivos Analíticos de Políticas Educativas: 22 (15). doi.org/10.14507/epaa.v22n15.2014

George, D., Mallery, P. (2003). SPSS for Windows step by step: A Simple Guide and Reference. 11.0 Update ( $4^{\mathrm{a}}$. ed.). Boston: Allyn \& Bacon.

INEE (2014) Perfil, parámetros e indicadores para docentes. Recuperado de http://servicioprofesionaldocente.sep.gob.mx/content/ba/docs/parametros_indicadores/Completo.pdf

INEE (2015a) Etapas, aspectos, métodos e instrumentos. Proceso de la evaluación del desempeño docente en la educación media superior. Recuperado de http://servicioprofesionaldocente.sep.gob.mx/content/ms/docs/2015/permanencia/etapas_aspectos/Etapas_Docentes_Desempeno_EMS.pdf

INEE (2015b) Perfiles, parámetros e indicadores. Recuperado de http://servicioprofesionaldocente.sep.gob.mx/content/ms/docs/2015/permanencia/PPI_DESEMPENO_EMS. pdf

Looney, J. (2011) Developing High-Quality Teachers: teacher evaluation for improvement. European Journal of Education, 46(4), 440-455.

Mancera, C. (2016). En el aula desempeño docente y aprendizaje. Nexos: sociedad, ciencia y literatura. Recuperado en agosto de 2017 de http://go.galegroup.com/ps/i.do? $\mathrm{p}=\mathrm{AONE} \& \mathrm{u}=$ pu\&id=GALE $\mid \mathrm{A} 459634715 \& \mathrm{v}=2.1 \& \mathrm{it}=\mathrm{r} \& \mathrm{sid}=$ summon\&authCount $=1$

Mas, O. (2012). Las competencias del docente universitario: la percepción del alumno, de los expertos y del propio protagonista. Revista de Docencia Universitaria, 10 (2), 299-318.

Montalvo, T. (2015, Febrero 11). En México se puede ser maestro de inglés... sin saber 
El perfil del docente competente de Humanidades y Ciencias Sociales en el bachillerato mexicano desde la perspectiva del alumno

ERIKA LÓPEZ RODRÍGUEZ

hablar inglés. Animal Político. Recuperado en 2017 de http://www.animalpolitico. com/2015/02/en-mexico-se-puede-ser-maestro-de-inglessin-saber-hablar-ingles/

Piñuel, J. (2002). Epistemología, metodología y técnicas del análisis de contenido. Universidad Complutense de Madrid. Estudios de Sociolingüística 3(1), 1-42.

Proyecto Tuning (2004). Proyecto Tuning América Latina. Recuperado de 2015 en http:// tuning.unideusto.org/tuningal/

RAE (2015). Diccionario de la lengua española (23ra Ed.) Madrid [en línea]. Recuperado de http://lema.rae.es/drae/?val=hacer

Rueda, M. (2009) La evaluación del desempeño docente: consideraciones desde el enfoque por competencias. Revista Electrónica de Investigación Educativa, 11(2), 1-16.

SEPa, (2017) Nuevo currículo de la Educación Media Superior. Campo disciplinar de Humanidades. Bachillerato General. Programa de las asignaturas del Bachillerato General. Nuevo Modelo Educativo. Recuperado de http://sems.gob.mx/es_mx/sems/ campos_disciplinares

SEPb, (2017) Nuevo currículo de la Educación Media Superior. Campo disciplinar de Ciencias Sociales. Bachillerato General. Programa de las asignaturas del Bachillerato General. Nuevo Modelo Educativo. Recuperado de http://sems.gob.mx/es_mx/sems/ campos_disciplinares

UNAM, (2016. Noviembre 15). Expertos analizan el modelo educativo. Boletín. Instituto de Investigaciones sobre la Universidad y la Educación. Recuperado de http://www. iisue.unam.mx/boletin/?p=4514

Villalobos, E.; Parés, I. (2007) Competencias Directivas del Docente Universitario. Revista Panamericana de Pedagogía, 10, 61-83.

Zabalza, M. (2003). Competencias docentes del profesorado universitario: calidad y desarrollo profesional. Madrid: Narcea.

Zambrano, R.; Meda, R.; Lara, B. (2005). Evaluación de profesores universitarios por parte de los alumnos mediante el Cuestionario de Evaluación del Desempeño Docente (CEDED). Revista de Educación y Desarrollo, 4, 63-69. 
